\title{
Stopping in ultrarelativistic nuclear collisions - results from NA49
}

\section{Marek KOWALSKI**}

Institute of Nuclear Physics, Cracow, Poland

E-mail: marek.kowalski@ifj.edu.pl

In this contribution we present a systematic study of the rapidity distributions of net protons as function of centrality in $\mathrm{Pb}+\mathrm{Pb}$ collisions at 158 and $40 \mathrm{GeV}$ per nucleon incident energy. The experimental nuclear collision data are compared to data from elementary nucleon-nucleon interactions.

European Physical Society Europhysics Conference on High Energy Physics July 16-22, 2009

Krakow, Poland

*Speaker.

${ }^{\dagger}$ For the NA49 Collaboration 


\section{Introduction}

It is now generally accepted that heavy ion collisions at ultra-relativistic energies result in a fireball of matter with high density and temperature. Such conditions prevail, when the incoming nucleons deposit enough of their kinetic energy in the reaction zone. A suitable observable to characterize the energy loss (stopping) of the incident nucleons is the longitudinal momentum of the net baryons after the collision. Results presented here come from the NA49 experiment at CERN SPS. The experimental layout, analysis method and the data selection method were described elsewhere $[1,2,3]$.

\section{Results}

In Fig. 1 net baryon rapidity distributions for different centralities in $\mathrm{Pb}+\mathrm{Pb}$ collisions at 40 $\mathrm{GeV} / \mathrm{N}$ and $158 \mathrm{GeV} / \mathrm{N}$ are shown. The centrality intervals range from the $0-5 \%$ most central events to the ensemble of events which populate the interval from 33.5 to $43.5 \%$. We note the approximate shape independence at $158 \mathrm{GeV} / \mathrm{N}$. At $40 \mathrm{GeV} / \mathrm{N}$ the shape shows a similar evolution as at AGS, although a dip at midrapidity persists at all energies.
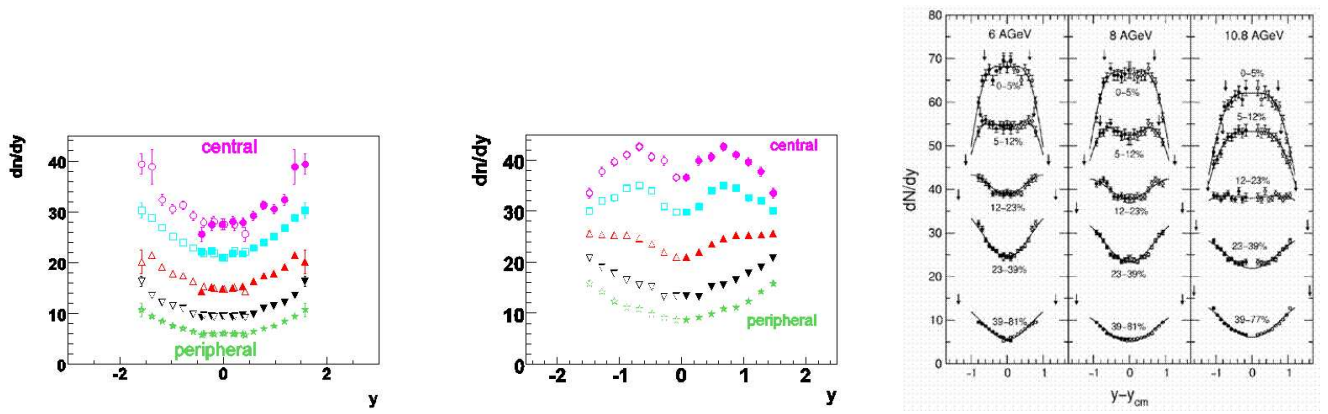

Figure 1: Net proton rapidity distribution for $158 \mathrm{GeV} / \mathrm{N}$ (left) and $40 \mathrm{GeV} / \mathrm{N}$ (middle) for different centralities. Full symbols shows the data, the open ones are reflected at midrapidity. The AGS data are shown on the right panel.

In Fig. 2 rapidity distributions for $\mathrm{p}, \mathrm{d}$ and ${ }^{3} \mathrm{He}$, normalized at midrapidity, are shown. The similarity of the distribution for $\mathrm{p}$ and $\mathrm{d}$ at $158 \mathrm{GeV} / \mathrm{c}$ indicates the coalescence as a possible source of ${ }^{3} \mathrm{He}$. Note a significant shape difference at $40 \mathrm{GeV} / \mathrm{N}$.
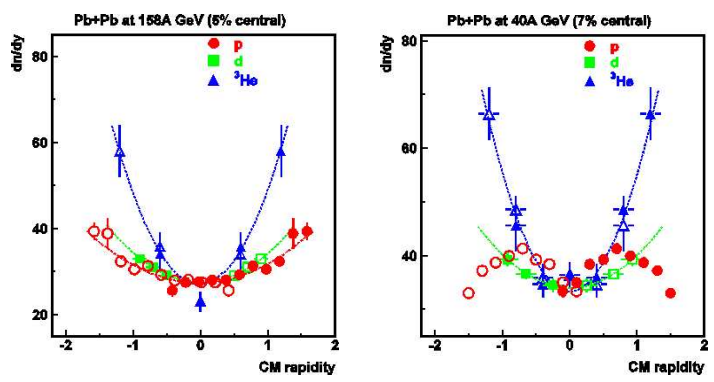

Figure 2: p, d and ${ }^{3} \mathrm{He}$ rapidity distributions at $158 \mathrm{GeV} / \mathrm{N}$ (left) and $40 \mathrm{GeV} / \mathrm{N}$ (right), normalized at midrapidity. 
Fig. 3 summarizes the most important conclusions from the study of proton production in $\mathrm{Pb}+\mathrm{Pb}$ and $\mathrm{p}+\mathrm{p}$ production at SPS energies. We compare the yield of net nucleons $(\mathrm{p}+\mathrm{n})$ as function of rapidity, normalized to the average number of wounded nucleons from $p+p$ interactions [4] with net protons from $\mathrm{Pb}+\mathrm{Pb}$ collisions at two centralities, the most central(upper) and the most peripheral (lower). We find that the $\mathrm{Pb}+\mathrm{Pb}$ and $\mathrm{p}+\mathrm{p}$ spectra are similar in shape and in yield. It seems that the effects due to secondary interactions of the participant nucleon happen mostly outside the experimental acceptance at higher energy. The multiple collision effects are coming out clearer at the lower energy (left column).

In view of the significant differences in the number of collisions per perticipating nucleon between $\mathrm{p}+\mathrm{p}$ and $\mathrm{Pb}+\mathrm{Pb}$ as well as in peripheral anc cental $\mathrm{Pb}+\mathrm{Pb}$ collisions, our findings suggests that multiple collisions have little influence on protons close to mid-rapidity. This is more pronounced at 158 than at $40 \mathrm{GeV} / \mathrm{N}$.
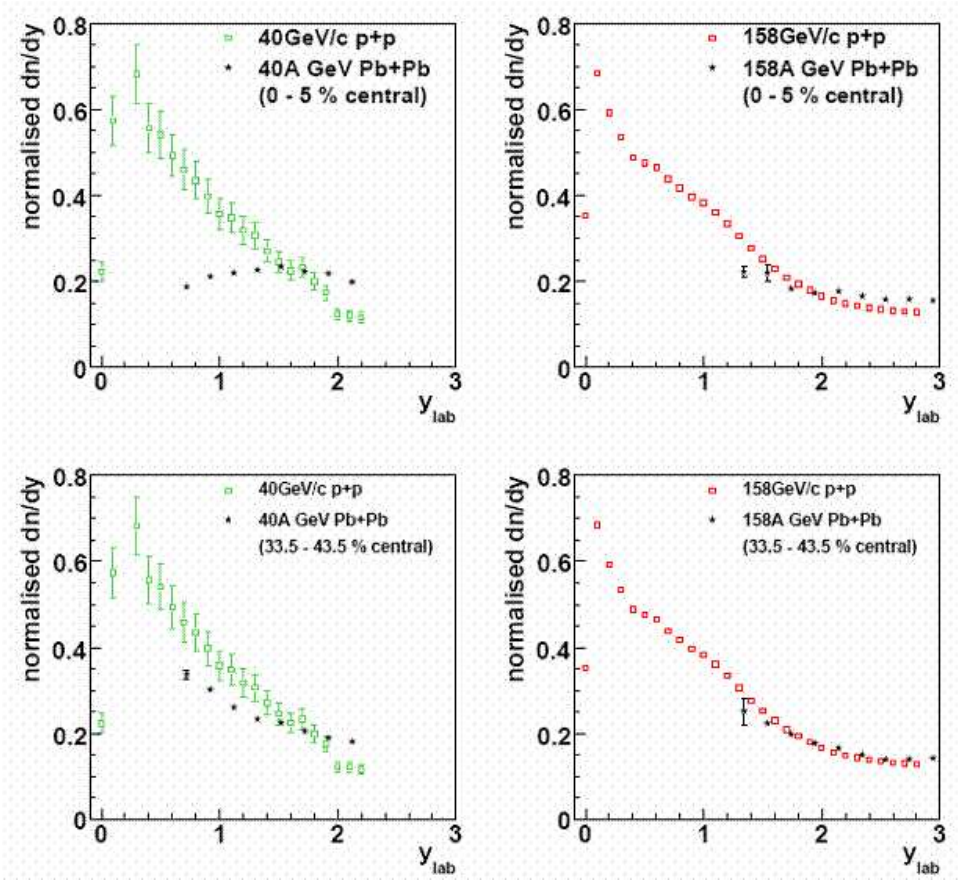

Figure 3: Normalized net proton rapidity distribution from $\mathrm{Pb}+\mathrm{Pb}$ at 40 (left) and $158 \mathrm{GeV} / \mathrm{N}$ (right) for the most central (upper) and the most peripheral (lower) centrality selection. The normalization constant is $\left\langle N_{w}>/ 2\right.$ to account for neutrons. Also shown, as a reference, are rapidity distributions of net nucleons $(\mathrm{p}+\mathrm{n})$ from $\mathrm{p}+\mathrm{p}$ interactions.

\section{References}

[1] S. V. Afanasiev et al., Nucl. Instrum. Meth. A430, 210 (1999)

[2] M. van Leeuwen, Ph.D. Thesis, NIKHEF, Amsterdam (2003); CERN EDMS Id 816033

[3] A. Laszlo, Ph.D. Thesis, KFKI Research Institute for Particle and Nuclear Physics, Budapest; CERN EDMS Id 942284

[4] T. Anticic et al. (NA49 Collaboration), preprint arXiv:0904.2708 [hep-ex] (2009) 